\title{
КВАЗИКЛАССИЧЕСКИЕ СПЕКТРАЛЬНЫЕ СЕРИИ ОПЕРАТОРА ШРЁДИНГЕРА НА ПОВЕРХНОСТЯХ В МАГНИТНОМ ПОЛЕ
}

\section{Р. В. Некрасов}

Рассматривается спектральная задача для оператора Шрёдингера заряженной частицы, удерживаемой на двумерной симметричной поверхности в однородном магнитном поле. Получены асимптотические серии спектра для случаев высоких и низких величин магнитного поля.

Библиография: 7 названий.

1. Постановка задачи. Стационарные состояния в квантовой задаче о движении электрона, удерживаемого на гладкой поверхности $S$ в магнитном поле $\mathbf{H}$, описываются уравнением Шрёдингера

$$
\widehat{H} \Psi=E \Psi .
$$

Здесь $\widehat{H}: L_{2}(S) \rightarrow L_{2}(S)$ - двумерный гамильтониан, выписанный ниже (оператор с символом, получающимся ограничением на кокасательное расслоение к $S$ функции Гамильтона $H(\mathbf{x}, \mathbf{p})=\frac{1}{2 \eta}\left(\mathbf{p}-\frac{e}{c} \mathbf{A}(x)\right)^{2}$ в объемлющем $\mathbb{R}^{6}$, где $e, \eta$ - заряд и масса частицы, $\mathbf{A}(x)$ - векторный потенциал магнитного поля).

Мы ограничиваемся поверхностями вращения $S$ в однородном поле с осью вращения $Z \uparrow \mathbf{H}$. Различных (с точностью до диффеоморфизма) связных поверхностей вращения $S$ всего четыре вида - плоскость, цилиндр, сфера, тор; из них мы будем рассматривать только компактные, т.е. диффеоморфные сфере $S^{2}$ или тору $T^{2}$.

Пусть $\theta$ - натуральный параметр на кривой вращения (ее длина), и $\phi-$ угол поворота этой кривой вокруг оси вращения. Эти две переменные параметризуют поверхность.

В координатах $\theta, \phi$ вместе с канонически сопряженными импульсами $p_{\theta}, p_{\phi}$ функция Гамильтона соответствующей механической системы имеет вид

$$
H\left(\theta, \phi, p_{\theta}, p_{\phi}\right)=\frac{p_{\theta}^{2}}{2 \eta}+\frac{1}{2 \eta}\left(\frac{p_{\phi}}{\rho(\theta)}+\frac{\eta w_{c} \rho(\theta)}{2}\right)^{2},
$$

где $w_{c}=e \mathrm{H} /(\eta c) \geqslant 0$ - циклотронная частота, $\rho(\theta)$ - расстояние от точки $\theta$ кривой вращения до оси $Z$.

Работа выполнена при финансовой поддержке фонда DFG-RAS, № 436 RUS 113/572.

(C) P. B. Некрасов, 2006 
Квантовый оператор Гамильтона стандартным образом получается из функции Гамильтона (1.1) заменой $\mathbf{p}^{2}$ на $-h^{2} \Delta$ и переменной $p_{\phi}$ на оператор $\hat{p}_{\phi}=-i h \frac{\partial}{\partial \phi}$ :

$$
\widehat{H}=-\frac{h^{2}}{2 \eta}\left\{\frac{1}{\rho(\theta)} \frac{\partial}{\partial \theta} \rho(\theta) \frac{\partial}{\partial \theta}+\frac{1}{\rho^{2}} \frac{\partial^{2}}{\partial \phi^{2}}\right\}-\frac{w_{c}}{2} i h \frac{\partial}{\partial \phi}+\frac{\eta w_{c}^{2}}{8} \rho^{2} .
$$

Компактность рассматриваемых поверхностей приводит к известному свойству дискретности спектра [1]-[3].

В настоящей работе исследуется квазиклассическая асимптотика при $h \rightarrow 0$ спектральной задачи для оператора Гамильтона $\widehat{H}$. Начнем с классического поведения системы.

\section{2. Классическая система. Топология разбиения фазового пространства}

на торы Лиувилля. Проекция $p_{\phi}$ момента движения на ось $Z$ вдоль поля сохраняется, что следует из симметрии вращения вокруг оси $Z$. Фазовое пространство $T^{*} S$ разбивается на инвариантные множества $H=$ const $=E, p_{\phi}=$ const $=M$. Такое разбиение, вообще говоря, имеет особенности; неособые слои представляют собой лиувиллевы торы (или объединение нескольких). В рассматриваемом ниже случае $w_{c} \neq 0$ особые слои могут содержать также окружности, изолированные точки равновесия или сингулярные критические множества [4]. Многообразие уровня $E, M$ обозначим $\mathscr{M}_{E, M}$. Опишем разбиение фазового пространства на такие замкнутые многообразия и режимы динамики на них.

Для самых простых поверхностей - стандартной сферы и стандартного тора вращения - разбиение плоскости $E, M$ на области, соответствующие различным режимам динамики, изображены на рис. 1, 2. Для точек областей $1,3,5,7$ имеется 1 лиувиллев тор $\mathscr{M}_{E, M}=L$ в фазовом пространстве $T^{*} S$, для областей $2,4,6,8$ уже 2 тора. Отмеченные точки имеют следующие координаты $(E, M): A=\left(0,-\frac{\eta w_{c} R^{2}}{2}\right)$, $B=\left(\frac{\eta w_{c}^{2} R^{2}}{2},+\frac{\eta w_{c} R^{2}}{2}\right)$. Ветвь параболы слева от $A$ дает правое периодическое вращение по экватору сферы, а ветвь справа - левое; дуга $A B$ отвечает также сепаратрисе в фазовом пространстве. Точка $O$ дает две изолированные точки покоя в полюсах, отрезок $(O, A]$ - целые окружности точек покоя, отрезок $(O, B]$ - окружности с вращением (Ларморовым). Линии перестройки проведены курсивом (на них сосредоточены все неустойчивые траектории - окружности, сингулярные критические множества).

Если сфера или тор не стандартные, но функция $\rho(\theta)$ для них устроена аналогично просто (количество критических точек минимально), то соответствующие рисунки (бифуркационные диаграммы) не изменяются. Если же критических точек больше, то анализ режимов несколько усложняется.

Именно, обозначим критические точки функции $\rho(\theta)$ как $\rho_{i}^{*}=\rho\left(\theta_{i}^{*}\right)$, и предположим так же, что все критические точки невырождены $\rho_{i}^{* \prime \prime} \neq 0$, выделим из них отдельно возрастающую последовательность минимумов $\rho_{1}<\rho_{2}<\cdots<\rho_{N^{\prime}}$ и убывающую последовательность локальных максимумов $\rho^{1}>\rho^{2} \ldots$

Всевозможные кривые вида

$$
2 \eta E=\left(\frac{M}{\rho_{i}^{*}}+\left(\frac{\eta w_{c} \rho_{i}^{*}}{2}\right)\right)^{2}, \quad i=1, \ldots, N
$$

или

$$
E=0, \quad E=M w_{c}
$$


разбивают плоскость $E, M$ на разные области, внутри которых многообразия $\mathscr{M}_{E, M}$ состоят из одинакового (возможно нулевого) числа торов Лиувилля. Движение в этих областях - условно периодическая намотка на один из торов Лиувилля $L \subset$ $\mathscr{M}_{E, M}$.

На границах этих областей (бифуркационных линиях) происходит следующее:

1) на прямых (2.2) многообразия $\mathscr{M}_{E, M}$ вырождаются в окружности. Окружности $\mathscr{M}_{0, M}, M<0$, для первой прямой состоят из точек покоя на расстоянии $\rho=\sqrt{-2 M /\left(\eta w_{c}\right)}$ от оси вращения, а окружности $\mathscr{M}_{M w_{c}, M}, M>0$, для второй прямой отвечают циклическим траекториям в фазовом пространстве, полностью расположенным на расстоянии $\rho=\sqrt{2 M /\left(\eta w_{c}\right)}$ от оси вращения (и движение является Ларморовым вращением с циклотронной частотой $\left.w_{c}\right)$;

2) на параболах (2.1) $\mathscr{M}_{E, M}$ могут содержать как все перечисленные компоненты (торы Лиувилля, окружности), так и сингулярные критические множества [4].

Если парабола (2.1) соответствует точке максимума $\rho_{i}^{*}=\rho^{j}$, то линия, отвечающая перестройкам торов Лиувилля, - это дуга параболы, заключенная между двумя точками касания с прямыми $(2.2)$, а если $\rho_{i}^{*}=\rho_{j}$ - точка минимума, то линия перестройки состоит из двух ветвей параболы, внешних по отношению к такой дуге.

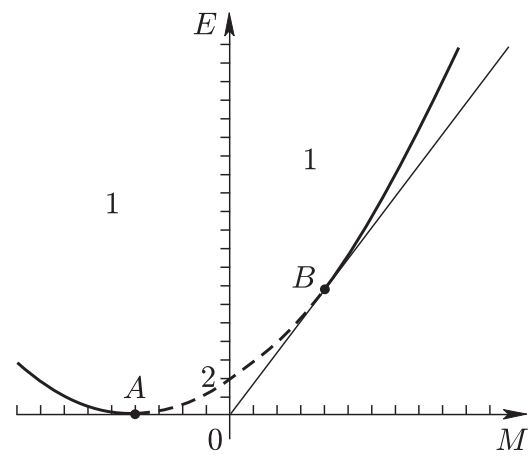

Рис. 1. Бифуркационная диаграмма для сферы.

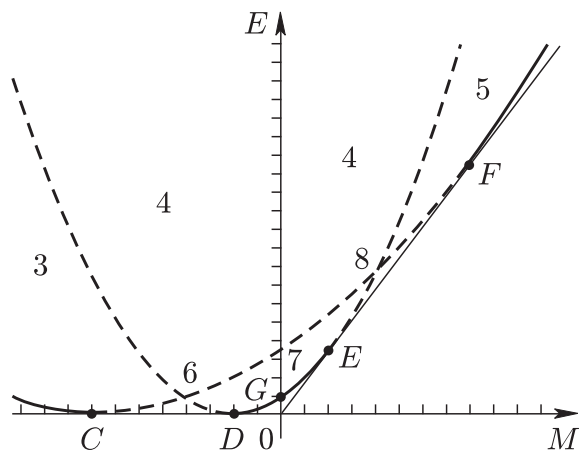

Рис. 2. Бифуркационная диаграмма для тора. 
3. Квазиклассическая асимптотика. Асимптотический спектр $E\left(h, w_{c}\right)$ оператора $\widehat{H}$ определяется из условий существования на лиувиллевых торах $L \subset \mathscr{M}_{E, M}$ (хотя бы одном) канонического оператора Маслова (так называемых условий квантования Бора-Зоммерфельда). Эти условия имеют вид

$$
\frac{1}{2 \pi h} \int_{\gamma_{i}}(p, d x)=n_{i}+\frac{\text { ind } \gamma_{i}}{4}, \quad n_{i} \in \mathbb{Z}, \quad n_{i} \sim \frac{1}{h}
$$

где $\gamma_{i}$ - базисные циклы на $L$, ind $\gamma_{i}$ - их индекс Маслова; $n_{i}$ - квантовые числа. Условия (3.1) - система двух уравнений для определения параметров $E\left(h, w_{c}\right)$, $M\left(h, w_{c}\right)$.

Теорема 1. Для любого значения $E\left(h, w_{c}\right)$, удовлетворяющего (3.1), найдется собственное число $\mathscr{E}\left(h, w_{c}\right)$ оператора $(1.2)$ такое, что $|E-\mathscr{E}|=O\left(h^{2}\right)$.

Более того, в любой компактной области $K$ плоскости $E, M$, не пересекающейся с бифуркационными линиями, эта ощенка равномерна:

$$
\operatorname{dist}(E, \operatorname{spec}(\widehat{H}))<C(K) h^{2},
$$

для любых $E, M \in K$ таких, что выполнено (3.1).

Применительно к заряженной частице на поверхности вращения $S$ на любом торе Лиувилля условие квантования для цикла вокруг оси $Z$ дает $M=m h, m \in \mathbb{Z}$, что совпадает с точным квантованием проекции момента $p_{\phi}$.

Условие (3.1) для второго фундаментального цикла дает трансцендентное соотношение на квантование второго интеграла движения - энергии:

$$
\frac{1}{\pi h} \int_{\theta_{\min }}^{\theta_{\max }} p_{\theta}(\theta) d \theta=l-\frac{\mu}{4}, \quad \text { где } \quad p_{\theta}(\theta)=\sqrt{2 \eta E-\left(\frac{M}{\rho(\theta)}+\frac{\eta w_{c} \rho(\theta)}{2}\right)^{2}}
$$

и $M=m h$, с учетом квантования $p_{\phi}$, а $\theta_{\max }, \theta_{\min }-$ соответственно максимальное и минимальное значения переменной $\theta$ для рассматриваемого тора $L \subset \mathscr{M}_{E, M}$, индекс Маслова $\mu=0, l=2 k, k \in \mathbb{N}$, если тор $L$ полностью накрывает $S=T^{2}$ при проекции $\left(p_{\theta}, p_{\phi}, \theta, \phi\right) \rightarrow(0,0, \theta, \phi)$, при этом состояния двукратно вырождены, в соответствии с двумя эквивалентными направлениями обмотки задаваемыми знаком $p_{\theta}$, и в остальных случаях $\mu=2, l \in \mathbb{N}$.

Трансцендентные соотношения на спектр можно разрешить для важных случаев высоких и низких полей (см. таблицу ниже). Оказывается, в этих выделенных случаях результаты определяются не всей геометрией поверхности вращения, а лишь топологией и небольшим количеством геометрических характеристик - главными кривизнами $\alpha_{j}=\frac{d^{2} \rho}{d \theta^{2}}\left(\theta_{j}\right), \frac{1}{\rho_{j}}$ в критических точках функции $\rho: j=1,2, \ldots, N^{\prime}$, а также радиусами кривизны $R_{1}, R_{2}$ полюсов (в случае сферической поверхности), длиной кривой вращения $L$ и средними $\rho_{s}^{-2}=\oint \rho^{-2}(\theta) d \theta$ (в случае тора). 
Теорема 2. Применение условий (3.1) для указанных случаев приводит $к$ следующей таблище результатов:

\begin{tabular}{|c|c|c|c|}
\hline Вид & $\begin{array}{c}\text { Энергия при } \\
\text { нулевом поле } \\
E_{l, m}(0) \bmod \left(\frac{m^{2}}{l^{2}}\right)\end{array}$ & $\begin{array}{c}\text { Энергия при } \\
\text { низких полях } \\
E_{l, m} \bmod \left(w_{c}^{2}\right) \\
w_{c} \rightarrow 0\end{array}$ & $\begin{array}{c}\text { Энергия при } \\
\text { высоких полях } \\
E_{l, m}\left(w_{c}\right) \quad \bmod (1) \\
w_{c} \rightarrow \infty\end{array}$ \\
\hline $\begin{array}{c}S \quad \text { диффео- } \\
\text { морфна сфере } \\
S \cong S^{2}\end{array}$ & $\begin{array}{l}\frac{h^{2} \pi^{2}}{2 \eta L^{2}}\left[l+|m|+\frac{1}{2}\right]^{2} \\
\quad l R_{1,2} \gg|m| L\end{array}$ & $E_{l, m}(0)+h w_{c} \frac{m}{2}$ & $\begin{array}{c}\frac{\eta w_{c}^{2} \rho_{i}^{2}}{8}+\frac{h w_{c}}{2} A_{l, m}^{i} \\
i=1,2, \ldots, N^{\prime} \\
h w_{c} A_{l, m}-\frac{h^{2}}{\eta R_{1,2}^{2}} B_{l, m}\end{array}$ \\
\hline $\begin{array}{c}S \text { - стандарт- } \\
\text { ная сфера } \\
S=S^{2}\end{array}$ & $\begin{array}{c}\frac{h^{2}}{2 \eta R^{2}}\left[l+|m|+\frac{1}{2}\right]^{2} \\
l \gg|m|\end{array}$ & $E_{l, m}(0)+h w_{c} \frac{m}{2}$ & $\begin{array}{c}h w_{c} A_{l, m}-\frac{h^{2}}{\eta R^{2}} B_{l, m} \\
\text { двукратное вырождение }\end{array}$ \\
\hline $\begin{array}{c}S \quad \text { диффео- } \\
\text { морфна тору } \\
S \cong T^{2} \\
\end{array}$ & $\begin{array}{c}\frac{h^{2}}{2 \eta}\left(\frac{4 \pi^{2} l^{2}}{L^{2}}+\frac{m^{2}}{\rho_{s}^{2}}\right) \\
|l| \rho_{1} \gg L|m| \\
\end{array}$ & $E_{l, m}(0)+h w_{c} \frac{m}{2}$ & $\begin{array}{c}\frac{\eta w_{c}^{2} \rho_{i}^{2}}{8}+\frac{h w_{c}}{2} A_{l, m}^{i}, \\
i=1,2, \ldots, N^{\prime}\end{array}$ \\
\hline $\begin{array}{c}S \text { - стандарт- } \\
\text { ный тор } \\
S=T^{2} \\
\end{array}$ & $\begin{array}{l}\frac{h^{2} \alpha_{1}^{2}}{2 \eta} l^{2}+\frac{h^{2} m^{2}}{2 \eta \rho_{s}^{2}} \\
|l| \gg L \alpha_{1}|m|\end{array}$ & $E_{l, m}(0)+h w_{c} \frac{m}{2}$ & $\frac{\eta w_{c}^{2} \rho_{1}^{2}}{8}+\frac{h w_{c}}{2} A_{l, m}^{1}$ \\
\hline
\end{tabular}

где

$$
\begin{gathered}
A_{l, m}^{i}=m+\sqrt{\alpha_{i} \rho_{i}}\left(l+\frac{1}{2}\right), \quad A_{l, m}= \begin{cases}\left(l+m+\frac{1}{2}\right), & m>0, \\
\left(l+\frac{1}{2}\right), & m<0,\end{cases} \\
B_{l, m}=\frac{1}{2}\left(l+\frac{1}{2}\right)^{2}+|m|\left(l+\frac{1}{2}\right) .
\end{gathered}
$$

В этой таблице первый столбец указывает вид поверхности. Второй столбец содержит данные об асимптотическом спектре, полученные в отсутствии магнитного поля $w_{c}=0$. Отметим, что при $m=0$ приведенные числа $E$ точно удовлетворяют условию Бора-Зоммерфельда. Кроме того, ясно, что относительная ошибка

$$
\frac{\triangle E_{l, m}}{E_{l, m}}<\frac{\triangle E}{E_{l, m}} \sim \frac{1}{\left(l^{2}+m^{2}\right)}
$$

$\left(\triangle E=C h^{2}\right.$ - расстояние от $E$ до спектра $\left.\widehat{H}\right)$ убывает с ростом $l, m \quad(|m| \leqslant O(|l|))$. Следующий столбец определяет производную по полю $\frac{d}{d w_{c}} E_{l, m}(0)=\frac{1}{2} m h$ линии асимптотического спектра, что совпадает с аналогичной производной линии точного. И, наконец, последняя колонка содержит всевозможные серии асимптотик по высоким полям, вычесленных с помощью условий Бора-Зоммерфельда. Полученные формулы, вообще говоря, не совпадают с асимптотиками точного спектра, так как они соответствуют областям в окрестности бифуркационных линий, где оценка (3.2) не действует. Однако эти бифуркационные линии отвечают устойчивым в линейном приближении точкам покоя и изотропным циклам, квантование которых методом комплексного ростка [7] дает, даже проще, тот же ответ (с точностью $о(h)$ или $o\left(w_{c}\right)$, если фиксировано $\left.h\right)$. Более того, из-за вырождения серий $A_{l, m}$ отвечающих полюсам, вычислены также поправки $\sim h^{2}$ к этим сериям. 
Видно, что выделяются серии квадратичных по $w_{c}$ асимптотик, соответствующих локальным минимумам $\rho_{i}$, и две серии линейных асимптотик в случае сферы $S^{2}$, соответствующие двум полюсам. Первый член линейных асимптотик - так называемые уровни Ландау; последний член $E^{(2)}=-\frac{h^{2}}{\eta R^{2}} B_{l, m}$, вообще говоря, отличается от аналогичного для асимптотики точного спектра, но, по-видимому, относительная ошибка определения $E^{(2)}$, как и в случае низких полей, с ростом $l,|m|$ уменьшается.

Следует заметить, что нумерация энергетических уровней в разных столбцах не совпадает (т.е. одной линии соответствуют разные квантовые числа в различных участках поля). Квантовые числа $l$ необходимо перенумеровывать после каждой перестройки торов Лиувилля.

Напоследок опишем вид асимптотической собственной функции $\Psi$, соответствующей фиксированному тору Лиувилля. Эта функция строится как действие канонического оператора Маслова $k$ (конструкция и свойства в [5], [6]) на константу: $\Psi=k[1]$.

Тот факт, что переменные разделяются дает о себе знать и при построении канонического оператора - его конструкция сводится к одномерной: $\Psi(\theta, \phi)=e^{i m \phi} \psi(\theta)$. Для функции $\psi$ внутри классически доступных областей имеем стандартное ВКБ решение, а вблизи границ - функции Эйри.

Если поверхность - тор, а проекция тора Лиувилля полностью его накрывает, то асимптотическое собственное значение двукратно вырождено,

$$
\psi_{1}(\theta)=\frac{1}{\sqrt{\rho(\theta) p(\theta)}} \cos \left(\frac{1}{h} \int_{\theta_{0}}^{\theta} p(\theta) d \theta\right), \quad \psi_{2}(\theta)=\frac{1}{\sqrt{\rho(\theta) p(\theta)}} \sin \left(\frac{1}{h} \int_{\theta_{0}}^{\theta} p(\theta) d \theta\right) .
$$

Во всех остальных случаях асимптотическая собственная функция внутри (на расстоянии порядка 1 от границ) классически доступной области имеет вид

$$
\psi(\theta)=\frac{1}{\sqrt{\rho(\theta) p(\theta)}} \cos \left(\frac{1}{h} \int_{\theta_{\min }}^{\theta} p(\theta) d \theta-\frac{\pi}{4}\right), \quad\{\theta\} \Subset\left[\theta_{\min }, \theta_{\max }\right],
$$

а вблизи границы $\theta_{b}$ (граничное $\theta: \theta_{\min }$ или $\theta_{\max }$ обозначаем как $\theta_{b \text { (oundary) }}$ ), т.е. при $\left|\theta-\theta_{b}\right| \lesssim 1$ асимптотическая функция приближенно выглядит как функция Эйри

$\psi_{b}(\theta) \approx \frac{h^{-\frac{2}{3}}}{\sqrt{\rho(\theta)}} A i\left(h^{-\frac{2}{3}} \beta_{\max / \min }^{\frac{1}{3}}\left(\theta-\theta_{b}\right)\right), \quad$ где $\quad \beta_{\max / \min }= \pm\left|\frac{2 \rho_{b}^{\prime}}{\rho_{b}}\left(\frac{M^{2}}{\rho_{b}^{2}}-\frac{w^{2} \eta^{2} \rho_{b}^{2}}{4}\right)\right|$,

a $\rho_{b}=\rho\left(\theta_{b}\right), \rho_{b}^{\prime}=\frac{d}{d \theta} \rho\left(\theta_{b}\right)$. Точность приближения пропорциональна $\left|\theta-\theta_{b}\right|^{2}$.

Функция $\psi_{b}$ асимптотически совпадает с (3.4) внутри классически доступной области, а вне равна 0, с любой точностью по малому $h: \psi_{b}\left(\theta \notin\left[\theta_{\min }, \theta_{\max }\right]\right)=o\left(h^{N}\right)$ $\forall N$.

Автор благодарит Шафаревича А. И. за постановку задачи и помощь в ее решении, а также Доброхотова С. Ю. за полезные замечания и дискуссии.

\section{СПИСОК ЦИТИРОВАННОЙ ЛИТЕРАТУРЫ}

[1] М. С. Агранович, “Эллиптические операторы на замкнутых многообразиях", Итоги науки и техники. Современные проблемы математики. Фундаментальные направления, 63, ВИНИТИ, М., 1990, 5-130. 
[2] М.А. Шубин, Псевдодифференциальные операторы и спектральная теория, Наука, M., 1978.

[3] B. Hellfer, "Theorie spectrale pour des operateurs globalement elliptiques", Asterisque, 1984.

[4] А. В. Болсинов, А. Т. Фоменко, Введение в топологию интегрируемых гамильтоновых систем, Наука, М., 1997.

[5] В.П. Маслов, М. В. Федорюк, Квазиклассическое приближение для уравнений квантовой механики, Наука, М., 1976.

[6] А.С. Мищенко, Б. Ю. Стернин, В.Е. Шаталов, Лагранжевъ многообразия и метод канонического оператора Маслова, Наука, М., 1978.

[7] С. Ю. Доброхотов, А. И. Шафаревич, "Квазиклассическое квантование инвариантных изотропных многообразий гамильтоновых систем", Топологические методы в теории гамильтоновых систем, Факториал, М., 1998.

Р. В. Некрасов

ИНЕСНЭК, г. Москва

E-mail: romun@mail.ru, logno@rambler.ru
Поступила в редакцию 26.03.2004

Исправленный вариант 08.06.2005 